\title{
On an HARQ-based coordinated multi-point network using dynamic point selection
}

\author{
Behrooz Makki ${ }^{*}$, Thomas Eriksson and Tommy Svensson
}

\begin{abstract}
This paper investigates the performance of coordinated multi-point (COMP) networks in the presence of hybrid automatic repeat request (HARQ) feedback. With an information theoretic point of view, the throughput and the outage probability of different HARQ protocols are studied for slow-fading channels. The results are compared with the ones obtained in the presence of repetition codes and basic $H A R Q$, or when there is no channel state information available at the base stations. The analytical and numerical results demonstrate the efficiency of the CoMP-HARQ techniques in different conditions.
\end{abstract}

\section{Introduction}

Coordinated multi-point (CoMP), also known as network multiple-input multiple-output (MIMO), is one of the most promising techniques for improving the data transmission efficiency of wireless cellular networks [1-4]. The main idea of a CoMP network is to allow geographically separated base stations (BSs) to cooperate in serving the users. The cooperation is achieved through high-speed backhaul links such that the users' data and channel state information (CSI) can be shared between the BSs. Theoretically, coordinated systems should outperform non-cooperative schemes. In practice, however, the performance gain of the CoMP networks largely depends on (1) the amount of CSI available at the transmitters (CSIT) and receivers (CSIR) and (2) the limited capacity of the backhaul links. This is the main motivation for studying the CoMP systems under limited CSI and backhaul capacity conditions, which has become a hot topic recently.

Performance of CoMP networks has been studied, e.g., by [5-10] under a perfect CSI assumption and by [11-14] in finite backhaul capacity conditions. The papers dealing with imperfect CSI in CoMP networks can be divided into two categories. The first group is the ones in which both the CSIT and the CSIR are assumed to be partially known [14-17]. In these works, the focus is on investigating the effect of training signals, providing imperfect

*Correspondence: behrooz.makki@chalmers.se

Department of Signals and Systems, Chalmers University of Technology, Gothenburg 412 96, Sweden
CSIR, on the network performance. The second group, on the other hand, is the papers in which, while the receivers are assumed to perfectly estimate the channels (perfect CSIR), the BSs have access to imperfect CSIT obtained by quantized CSI feedback [18-30]. Here, it is concentrated on the effects of the CSIT quantization and the quantized CSIT error is normally modeled as Gaussian noise. However, some other CSI quantization schemes have been summarized in [21-28] as well. Finally, to study the effect of BS synchronization, [27-30] have considered the cases where the BSs are provided with quantized CSI of the channel phase, while the amplitudes are perfectly fed back. Some of the most important conclusions drawn from these works are as follows:

- Compared to non-cooperative systems, the cooperation-based schemes suffer more from inaccurate CSIR. That is, the CoMP systems need larger number of pilot symbols than single-cell transmission to make use of its potential [14-17]. Therefore, the CoMP networks are more appropriate for low-mobility communication setups, where the channels change slowly and can be estimated accurately.

- The presence of phase ambiguity and asynchronous data transmission can degrade the system performance severely and even make it worse than a non-CoMP model [28-30]. Particularly, an important phase ambiguity source, not considered by [28-30], is phase-noise and frequency offset $[31,32]$ which makes the synchronization very difficult.

\section{Springer}

(c) 2013 Makki et al. licensee Springer. This is an Open Access article distributed under the terms of the Creative Commons Attribution License (http://creativecommons.org/licenses/by/2.0), which permits unrestricted use, distribution, and reproduction in any medium, provided the original work is properly cited. 
- For realistic results, simple cooperation schemes should be considered, where not only the amount of backhaul capacity and feedback resources is minimized but also the implementation complexity is as low as possible.

These points are the main motivations for our paper. Here, the goal is to develop a simple cooperation scheme for low-mobility CoMP networks which leads to minimum feedback requirement, acceptable backhauling resources, and affordable implementation complexity. Therefore, we propose that hybrid automatic repeat request (HARQ) feedback is selected and, at every moment, each user is served by a single BS, while the serving BSs are switched in the successive time slots. The reasons for our selection are

- HARQ is a technique in the data link layer already provided in many wireless protocols, e.g., IEEE 802.11n [33], IEEE 802.16e [34], and 3GPP LTE [35]. Hence, it needs no new additional design which introduces it as a cost- and complexity-efficient approach.

- From an information theory point of view, HARQ is a sequential feedback approach; in each

(re)transmission round, only 1-bit feedback is sent by the users which, compared to the quantized CSI schemes reporting all corresponding fading coefficients of the channel, reduces the feedback load substantially. Moreover, there is no quantized CSI feedback to be shared between the BSs, decreasing the backhauling requirements (Meanwhile, the HARQ feedback bits are shared between the BSs).

- In the proposed scheme, each user is served by only one BS in each time slot. Therefore, there is no need to synchronize the BSs, which decreases the implementation complexity substantially.

In summary, the proposed scheme achieves some advantages related to coordinated data transmission but can still avoid many problems that may limit the practical implementation of CoMP networks.

The results are of particular interest when we remember that, although HARQ schemes have been widely studied in single-user [36-41] and MIMO systems with asymptotically high signal-to-noise ratios (SNRs) [42-47], there are very few results for CoMP-HARQ [48]. Specifically, reviewing the third generation partnership project (3GPP) reports, e.g. [49], there are many aspects of the CoMPHARQ that have not been studied yet. Therefore, the final conclusions of the paper should be useful for the people involved in limited feedback issues in CoMP network standardization.

As described both theoretically and numerically, the proposed scheme leads to considerable performance improvement in terms of system throughput and the user outage probability. Particularly, the BS cooperation makes it possible to combine the advantages of fast-fading channels, having a large diversity gain, with the potential for accurate channel estimation in slow-fading channels. For sufficiently large number of users, the proposed CoMPHARQ scheme can be modeled as a collection of singleuser interference-free networks experiencing a modified SNR. Moreover, to have a positive multiplexing gain, the number of retransmissions should be scaled with the transmission power. Finally, compared to code-combining HARQ protocols, the diversity combining schemes are preferable at low powers, because they lead to the same throughput and outage probability with less complexity at the encoders and decoders.

\section{System model}

Consider a CoMP communication setup consisting of $N$ geographically distributed BSs and $K$ users, with $K \geq N$. The BSs are connected via delay-free backhaul links such that the users' data can be shared between the BSs. The BSs are limited to a peak power constraint $P$. Let $h_{j, i}$ be the channel coefficient between the $j$-th BS and the $i$-th user. Also, define the channel gains $g_{j, i} \doteq\left|h_{j, i}\right|^{2}$. We study the low-mobility, also called slow-fading, scenario where the channel gains remain constant in a fading block, determined by the channel coherence time $L_{\mathrm{c}}$, and then change independently to other values according to the probability density functions (pdfs) $f_{g_{j, i}}($.). The simulation results are given for both homogenous and heterogenous links. However, to be more tractable, the analytical results are presented for homogenous links where the channels experience the same fading pdfs. Note that considering the same pdfs does not mean that the channel realizations are the same in a time slot, as they have independent random values. However, for ergodic channels, the same pdf indicates that in the long-run, i.e., over infinitely many time slots, they experience the same behavior. Also, extension of the results for heterogenous networks is straightforward.

The length of the fading block, $L_{\mathrm{c}}$, is assumed to be so long that many packets ${ }^{\mathrm{a}}$ are transmitted in a single fading block. As a result, the channel gains can be assumed to be perfectly known by the receivers [18-30,36-41]. On the other hand, there is no CSI available at the BSs (CSIT), except the HARQ feedback bits. The complex white Gaussian noises added at the receivers are supposed to have distributions $\mathcal{C N}(0,1)$. Finally, the results are presented in natural logarithm basis, and the throughput is obtained in nats per channel use (npcu).

\subsection{Data transmission model}

A maximum of $M$ HARQ-based retransmission rounds are considered, i.e., each codeword is (re)transmitted a 
maximum of $M+1$ times. In each fading block, $N$ users are selected randomly ${ }^{\mathrm{b}}$. From a specific user perspective, the user receives the data from one BS in each time slot. Then, in the next slot, the serving BSs are switched and the user is served by another BS. The BS switching is done independently of whether new codewords are going to be sent to the users or the previous messages should be retransmitted in an HARQ-based fashion. By this technique, the HARQ protocol has a large diversity gain compared to the non-CoMP case.

Remark 1. Four different schemes have been considered for the CoMP networks in the 3GPP community during Release 11 [50,51]: (1) joint transmission, (2) dynamic point selection (DPS), (3) dynamic point blanking, and (4) coordinated scheduling/beamforming. The proposed scheme belongs to the DPS approach of the CoMP networks where the transmission points are varied according to the considered cooperation rules.

Here, although some discussions about the basic ARQ and repetition codes are presented, the main focus is on two stop-and-wait HARQ protocols:

1) Repetition time diversity (RTD). This scheme belongs to the diversity combining category of HARQ protocols [52] where the same data is repeated in the (re)transmission rounds and, in each round, the receiver performs maximum ratio combining (MRC) of all received signals.

2) Incremental redundancy (INR). The INR belongs to the category of code combining protocols [52]. Here, a codeword is sent with an aggressive rate in the first round. Then, if the user cannot decode the initial codeword, further parity bits are sent in the next retransmission rounds and in each round, the receiver decodes the data based on all received signals.

In harmony with, e.g., [36-41], we study the system performance for the complex Gaussian codes which have been proved to be optimal for power-limited data transmission in fading AWGN channels with long codewords ([53], Chapter 9). The same approach as in, e.g., [54], can be used to extend the results to the case with, e.g., low-density parity-check codes.

\subsection{Figures of merit}

We consider two, namely, outage probability and longterm throughput, evaluation yardsticks. A user outage probability is the probability of the event that the data cannot be decoded by the user when all retransmission rounds are used. The long-term throughput, on the other hand, is defined as follows. Let the total number of channel uses and the number of successfully decoded information nats up to the end of the $k$-th slot be $\tau^{(k)}$ and $Q^{(k)}$, respectively. Then, the long-term throughput [36-41] is defined as

$$
\eta=\lim _{k \rightarrow \infty} \frac{Q^{(k)}}{\tau^{(k)}}=\frac{\mathbf{E}\{\tilde{Q}\}}{\mathbf{E}\{\tilde{\tau}\}},
$$

where $\mathbf{E}\{$.$\} is the expectation operator and \tilde{Q}$ and $\tilde{\tau}$ are the number of successfully decoded information nats and the number of channel uses in a fading block, respectively. Here, the continuous communication model is considered, where the users are always actively requesting new information. In this case, all of the channel uses during a fading block are used. Therefore, defining $\tilde{R}$ as the achievable rate random variable of the HARQ protocols in a fading block, the long-term throughput is rephrased as

$$
\eta=\frac{\mathbf{E}\left\{L_{\mathrm{c}} \tilde{R}\right\}}{L_{\mathrm{c}}}=\mathbf{E}\{\tilde{R}\},
$$

i.e., the average rate [37-40].

\section{Performance analysis for the RTD HARQ protocol}

In this section, we derive the throughput and outage probability of the network in the presence of RTD HARQ protocol. The simulation results for the RTD are summarized in the figures presented in Section 6.

With no loss of generality, assume that the new data transmission of the $k$-th user is started from the $k$-th BS. Also, let us focus on a specific, say the first, user while the final throughput is obtained by summation on all users throughput. In this way, the signal-to-interference-andnoise ratio (SINR) received at the first user in the $m$-th (re)transmission round is

$$
\gamma^{m}=\frac{P g_{m, 1}}{1+P \sum_{j=1, j \neq m}^{N} g_{j, 1}} .
$$

Here, (3) is based on the fact that starting from the first BS, the user is served by the $m$-th BS in the $m$ th (re)transmission round. Considering Rayleigh fading channels $f_{g_{j, i}}(g)=\lambda e^{-\lambda g}, g \geq 0, \forall i, j$, on which we focus, the SINR cumulative distribution function (cdf) is obtained as

$F_{\gamma^{m}}(x)=\operatorname{Pr}\left\{\frac{P g_{m, 1}}{1+P \sum_{j=1, j \neq m}^{N} g_{j, 1}} \leq x\right\} \stackrel{(a)}{=} 1-\frac{e^{-\frac{\lambda}{P} x}}{(1+x)^{N-1}}, x \geq 0$,

where $(a)$ is found by integration on the gains pdfs and $\lambda$ represents the fading parameter determined by the path loss and shadowing between the BSs and the terminals ${ }^{\mathrm{c}}$.

Using MRC at the receiver, the equivalent received SINR at the end of the $m$-th (re)transmission round of the RTD is $\gamma^{(m)}=\sum_{n=1}^{m} \gamma^{n}$. Also, as the same data is repeated in the retransmission rounds of the RTD, the equivalent data rate at the end of the $m$-th round is $R^{(m)}=\frac{Q}{m L}=\frac{R}{m}$ 
where $Q$ is the number of information nats considered for the initial codeword, $L$ denotes the codewords length, and $R=\frac{Q}{L}$ represents the initial codeword rate.

The data is decoded at the end of the $m$-th round if (1) it has not been decoded before and (2) retransmitting the data at the $m$-th round, the channel SINR, accumulated after MRC, supports the data rate. In this way, the achievable data rate random variable for the RTD protocol is obtained as

$\tilde{R}^{\mathrm{RTD}}=\left\{\begin{array}{l}\frac{R}{m} \text { if } \log \left(1+\gamma^{(m-1)}\right)<R \leq \log \left(1+\gamma^{(m)}\right), \gamma^{(m)}=\sum_{n=1}^{m} \gamma^{n} \\ 0 \text { if } R>\log \left(1+\gamma^{(M+1)}\right)\end{array}\right.$

where $\gamma^{0} \doteq 0$. Here, (5) follows from the fact that with accumulated SINR $x$ at the end of the $m$-th round the maximum decodable data rate is $\frac{1}{m} \log (1+x)$ if the codeword is repeated $m$ times. In this way, the long-term throughput for the first user is

$$
\begin{aligned}
\eta_{1}^{\mathrm{RTD}} & =\sum_{m=1}^{M+1} \frac{R}{m} \operatorname{Pr}\left\{\log \left(1+\gamma^{(m-1)}\right)<R \leq \log \left(1+\gamma^{(m)}\right)\right\} \\
& =\sum_{m=1}^{M+1} \frac{R}{m}\left(F_{\gamma^{(m-1)}}\left(e^{R}-1\right)-F_{\gamma^{(m)}}\left(e^{R}-1\right)\right)
\end{aligned}
$$

where $F_{\gamma^{(m)}}$ is the cdf of auxiliary variable $\gamma^{(m)}$. Hence, as the packet starts from each BS with probability $\frac{1}{N}$ and the network is homogenous, the system long-term throughput is found as

$$
\begin{aligned}
\eta^{\mathrm{RTD}} & =N \eta_{1}^{\mathrm{RTD}} \\
& =N \sum_{m=1}^{M+1} \frac{R}{m}\left(F_{\gamma^{(m-1)}}\left(e^{R}-1\right)-F_{\gamma^{(m)}}\left(e^{R}-1\right)\right) .
\end{aligned}
$$

Here, (7) gives the users expected achievable rates for different message decoding conditions. Moreover, (7) indicates that the performance of the HARQ protocol improves as the number of retransmissions increases (as seen in the following, the throughput is scaled with the number of retransmission rounds at most logarithmically). Moreover, the user outage probability is

$$
\begin{aligned}
\operatorname{Pr} \text { \{outage }_{i}^{\mathrm{RTD}} & =\operatorname{Pr}\left\{R>\log \left(1+\gamma^{(M+1)}\right)\right\} \\
& =F_{\gamma^{(M+1)}}\left(e^{R}-1\right), \forall i
\end{aligned}
$$

The optimal parameter $R$, in terms of, e.g., throughput, can be found numerically. Finally, bounds of the throughput and comparisons between the RTD and other schemes are presented in Section 5.

\section{Performance analysis for the INR HARQ protocol}

As mentioned before, the INR protocol is based on an aggressive codeword transmission in the first round, sending further parity bits in the retransmissions and combining all received representations of the signal at the receiver. Therefore, the results of, e.g., [36-39,41] can be used to find the achievable rate random variable of the first user with packet transmission starting from the first BS as

$\tilde{R}^{\mathrm{INR}}=\left\{\begin{array}{l}\frac{R}{m} \text { if } \sum_{n=1}^{m-1} \log \left(1+\gamma^{n}\right)<R \leq \sum_{n=1}^{m} \log \left(1+\gamma^{n}\right) \\ 0 \text { if } R>\sum_{n=1}^{M+1} \log \left(1+\gamma^{n}\right) .\end{array}\right.$

Here, (9) is based on the fact that with SINR $\gamma^{n}$ in the $n$-th, $n=1, \ldots, m$, time slot the maximum decodable data rate is $\frac{1}{m} \sum_{n=1}^{m} \log \left(1+\gamma^{n}\right)$ if different codewords are sent in each round. In this way, the first user long-term throughput is found as

$$
\eta_{1}^{\mathrm{INR}}=\sum_{m=1}^{M+1} \frac{R}{m} \operatorname{Pr}\left\{\sum_{n=1}^{m-1} \log \left(1+\gamma^{n}\right)<R \leq \sum_{n=1}^{m} \log \left(1+\gamma^{n}\right)\right\}
$$

and the system long-term throughput is

$\eta^{\mathrm{INR}}=N \sum_{m=1}^{M+1} \frac{R}{m} \operatorname{Pr}\left\{\sum_{n=1}^{m-1} \log \left(1+\gamma^{n}\right)<R \leq \sum_{n=1}^{m} \log \left(1+\gamma^{n}\right)\right\}$.

According to (7) and (11), the only difference between the RTD and INR protocols is in the achievable rate terms where $\log \left(1+\sum_{n=1}^{m} \gamma^{n}\right)$ of the RTD is replaced by $\sum_{n=1}^{m} \log \left(1+\gamma^{n}\right)$ in the INR (Further comparisons between the RTD and INR protocols are presented in Section 5). Again, $R$ is optimized to maximize the throughput for each transmission power. Also, the user outage probability is obtained by

$$
\operatorname{Pr}\{\text { outage }\}_{i}^{\mathrm{INR}}=\operatorname{Pr}\left\{R>\sum_{n=1}^{M+1} \log \left(1+\gamma^{n}\right)\right\}, \forall i .
$$

In Section 5, we use (10) to (12) to analyze the throughput at asymptotically low and high powers, and evaluate the effect of BS cooperation on the performance of the network.

\section{On the performance of the proposed schemes}

In this section, we derive performance bounds for various cases and compare the performance of different schemes operating in the CoMP scenario.

\subsection{Performance bounds}

This subsection presents performance bounds/approximations for the throughput and outage probability of the proposed CoMP-ARQ approach. Theorem 1 shows 
that the performance of the considered protocols becomes bounded if the number of retransmission rounds is finite.

Theorem 1. With a fixed number of retransmissions, the throughput achieved by the considered CoMP-HARQ schemes is upper bounded even if the transmission power goes to infinity.

Proof. The theorem is proved for the INR protocol which, as stated in the following, results in higher throughput compared to the RTD. The throughput upper bound is found based on the following (in)equalities:

$$
\begin{aligned}
\eta^{\mathrm{INR}} \stackrel{(b)}{=} & N \sum_{m=1}^{M} \frac{R}{m(m+1)} \operatorname{Pr}\left\{\sum_{n=1}^{m} \log \left(1+\gamma^{n}\right) \geq R\right\} \\
& +\frac{N R}{M+1} \operatorname{Pr}\left\{\sum_{n=1}^{M+1} \log \left(1+\gamma^{n}\right) \geq R\right\} \\
& \stackrel{(c)}{\leq} N \sum_{m=1}^{M} \frac{\mathbf{E}\left\{\sum_{n=1}^{m} \log \left(1+\gamma^{n}\right)\right\}}{m(m+1)}+\frac{N \mathbf{E}\left\{\sum_{n=1}^{M+1} \log \left(1+\gamma^{n}\right)\right\}}{M+1} \\
& \stackrel{(d)}{\leq} \frac{N}{N-1} \sum_{m=0}^{M} \frac{1}{m+1} \stackrel{(e)}{\leq} \frac{N}{N-1}(1+\log (M+1)) .
\end{aligned}
$$

Here, $(b)$ is obtained by some manipulation on (11) and (c) comes from the Markov's inequality, $\operatorname{Pr}(X \geq x) \leq \frac{\mathbf{E}(X)}{x}$ [55]. Then, (d) follows from

$$
\begin{aligned}
\Lambda & =\mathbf{E}\left\{\sum_{n=1}^{m} \log \left(1+\gamma^{n}\right)\right\}=m \mathbf{E}\left\{\log \left(1+\gamma^{n}\right)\right\} \\
& =m \int_{0}^{\infty} \log (1+x) f_{\gamma^{n}}(x) \mathrm{d} x \\
& \stackrel{(f)}{=} m \int_{0}^{\infty}\left(1-F_{\gamma^{n}}\left(e^{z}-1\right)\right) \mathrm{d} z \stackrel{(g)}{\leq} m \int_{0}^{\infty} e^{-(N-1) z} \mathrm{~d} z=\frac{m}{N-1},
\end{aligned}
$$

where $(f)$ is obtained by variable transform $\log (1+x)=$ $z$ and partial integration and $(g)$ is based on (4) and $e^{-\frac{\lambda}{P} x} \leq 1$. Finally, $(e)$ in (13) follows from $\sum_{m=0}^{M} \frac{1}{m+1} \leq$ $1+\log (M+1)$ which is obtained by Riemann integral and the fact that $\frac{1}{x}$ is a decreasing function ${ }^{\mathrm{d}}$.

Intuitively, both the signal and the interference powers grow linearly with $P$. Hence, with high transmission power, the system becomes interference-limited and, according to (4), the users received SINR is bounded. In other words, the theorem states that: (1) the multiplexingz gain of the proposed CoMP scheme, defined as $\rho=\lim _{P \rightarrow \infty} \frac{\eta}{\log (P)}$ [56], is zero if a fixed number of retransmission rounds are considered for the HARQ protocols. (2) To have a positive multiplexing gain, a necessary condition is to scale the number of retransmission rounds with the transmission power. (3) Also, according to (13), the throughput is scaled with the number of retransmission rounds at most logarithmically. Finally, denoting the channel instantaneous capacity by $C$, we can use $(b)$ in (13) to write

$$
\begin{aligned}
\eta^{\mathrm{INR}} & \stackrel{(h)}{\leq} \\
& N R\left(\sum_{m=1}^{M} \frac{e^{-\frac{1}{m}} \mathbf{E}\left\{\left(\frac{1}{m} \sum_{n=1}^{m} \log \left(1+\gamma^{n}\right)\right)^{\frac{1}{R}}\right\}}{m(m+1)}\right. \\
& \left.+\frac{e^{-\frac{1}{M+1}} \mathbf{E}\left\{\left(\frac{1}{M+1} \sum_{n=1}^{M+1} \log \left(1+\gamma^{n}\right)\right)^{\frac{1}{R}}\right\}}{M+1}\right) \\
& \stackrel{(i)}{\leq} N R \mathbf{E}\left\{C^{\frac{1}{R}}\right\}\left(\sum_{m=1}^{M} \frac{e^{-\frac{1}{m}}}{m(m+1)}+\frac{e^{-\frac{1}{M+1}}}{M+1}\right) \\
& \stackrel{(j)}{\leq} N R \mathbf{E}\left\{C^{\frac{1}{R}}\right\}(\xi+1)
\end{aligned}
$$

which indicates that for sufficiently high initial transmission rate, where $\mathbf{E}\left\{C^{\frac{1}{R}}\right\} \rightarrow 1$, the throughput scales with the initial rate $R$ at most linearly. Note that (h) in (15) is obtained with the same procedure as in $(b)$ of (13) and then implementation of the exponential Chebyshev's inequality, $\operatorname{Pr}(X \geq x) \leq e^{-t x} \mathrm{E}\left(e^{t X}\right), \forall t>0$ [55]. Also, (i) follows from the fact that $\frac{1}{m} \sum_{n=1}^{m} \log \left(1+\gamma^{n}\right) \leq C$, i.e., the maximum decodable rates is less than the channel instantaneous capacity $C$, and $(j)$ comes from $\frac{e^{-\frac{1}{M+1}}}{M+1} \leq 1$ and $\sum_{m=1}^{M} \frac{e^{-\frac{1}{m}}}{m(m+1)} \leq \xi$ where $\xi$ is the Euler's number. Finally, the same qualitative conclusions are valid for the RTD protocol.

Theorem 2. For Rayleigh fading channels, the proposed CoMP-HARQ network can be modeled/underestimated by $N$ interference-free single-user networks having a modified transmission SNR.

Proof. With the same procedure as in (13), the RTD- and INR-based throughput, i.e., (7) and (11), can be rewritten as

$$
\begin{array}{r}
\eta^{\mathrm{RTD}}=N \sum_{m=1}^{M} \frac{R}{m(m+1)} \operatorname{Pr}\left\{\log \left(1+\sum_{n=1}^{m} \gamma^{n}\right) \geq R\right\} \\
+\frac{N R}{M+1} \operatorname{Pr}\left\{\log \left(1+\sum_{n=1}^{M+1} \gamma^{n}\right) \geq R\right\}
\end{array}
$$

$$
\begin{array}{r}
\eta^{\mathrm{INR}}=N \sum_{m=1}^{M} \frac{R}{m(m+1)} \operatorname{Pr}\left\{\sum_{n=1}^{m} \log \left(1+\gamma^{n}\right) \geq R\right\} \\
+\frac{N R}{M+1} \operatorname{Pr}\left\{\sum_{n=1}^{M+1} \log \left(1+\gamma^{n}\right) \geq R\right\} .
\end{array}
$$

Moreover, from (4), we can show that the auxiliary random variable $\gamma^{n}$ is dominated ${ }^{\mathrm{e}}$ by the random variable $\omega^{n}$ which follows the cdf $f^{f}$

$$
F_{\omega^{n}}(x)=1-e^{-\left(\frac{\lambda}{P}+N-1\right) x} .
$$


Therefore, using (16), (17), and the fact that $F_{\gamma^{n}}(x) \leq$ $F_{\omega^{n}}(x), \forall x$, i.e., $\operatorname{Pr}\left\{\gamma^{n} \geq x\right\} \geq \operatorname{Pr}\left\{\omega^{n} \geq x\right\}, \forall x$, we have

$$
\begin{aligned}
\eta^{\mathrm{RTD}} \geq & N \sum_{m=1}^{M} \frac{R}{m(m+1)} \operatorname{Pr}\left\{\log \left(1+\sum_{n=1}^{m} \omega^{n}\right) \geq R\right\} \\
& +\frac{N R}{M+1} \operatorname{Pr}\left\{\log \left(1+\sum_{n=1}^{M+1} \omega^{n}\right) \geq R\right\} \\
\stackrel{(k)}{=} & N \sum_{m=1}^{M} \frac{R}{m(m+1)} \operatorname{Pr}\left\{\log \left(1+\bar{P} \sum_{n=1}^{m} \bar{\omega}^{n}\right) \geq R\right\} \\
& +\frac{N R}{M+1} \operatorname{Pr}\left\{\log \left(1+\bar{P} \sum_{n=1}^{M+1} \bar{\omega}^{n}\right) \geq R\right\}=\eta^{\mathrm{RTD}, \mathrm{SU}}
\end{aligned}
$$

$$
\begin{aligned}
\eta^{\mathrm{INR}} \geq & N \sum_{m=1}^{M} \frac{R}{m(m+1)} \operatorname{Pr}\left\{\sum_{n=1}^{m} \log \left(1+\omega^{n}\right) \geq R\right\} \\
& +\frac{N R}{M+1} \operatorname{Pr}\left\{\sum_{n=1}^{M+1} \log \left(1+\omega^{n}\right) \geq R\right\} \\
\stackrel{(k)}{=} & N \sum_{m=1}^{M} \frac{R}{m(m+1)} \operatorname{Pr}\left\{\sum_{n=1}^{m} \log \left(1+\bar{P} \bar{\omega}^{n}\right) \geq R\right\} \\
& +\frac{N R}{M+1} \operatorname{Pr}\left\{\sum_{n=1}^{M+1} \log \left(1+\bar{P} \bar{\omega}^{n}\right) \geq R\right\}=\eta^{\mathrm{INR}, \mathrm{SU}} .
\end{aligned}
$$

Here, $(k)$ in (19) and (20) is obtained by defining $\bar{\omega}^{n}$ : $F_{\bar{\omega}^{n}}(x)=1-e^{-x}$ and the equivalent SNR $\bar{P}=\frac{P}{\lambda+(N-1) P}$, i.e., by appropriate scaling of the fading pdf and the transmission SNR. Also, $\eta^{\mathrm{RTD}, \mathrm{SU}}$ and $\eta^{\mathrm{INR}, \mathrm{SU}}$ denote the throughput achieved by the RTD and INR protocols, respectively, in the equivalent channel model of (18). In words, (19) and (20) imply that the CoMP-HARQ network performance can be underestimated by $N$ interferencefree single-input single-output (SISO) Rayleigh fading channels with fading coefficient $h \sim \mathcal{C N}(0,1), \bar{\omega}^{n}=$ $|h|^{2}$, and transmission SNR $\bar{P}=\frac{P}{\lambda+(N-1) P}$. Interestingly, the approximation $F_{\gamma^{n}} \simeq F_{\omega^{n}}$ becomes very tight for moderate/high values of $N$. Thus, the collection of SISO channels becomes an accurate model for the proposed CoMP-HARQ network, as stated in the theorem.

One of the benefits of Theorem 2 is that there are closed-form/approximate expressions for the probability terms of (19) and (20) such as

$$
\operatorname{Pr}\left\{\log \left(1+\bar{P} \sum_{n=1}^{m} \bar{\omega}^{n}\right) \geq R\right\}=1-V\left(\frac{e^{R}-1}{\bar{P}}, m\right),
$$

where $V(x, y)$ is the normalized incomplete Gamma function (please see [57] for more details about (21)).

\subsection{Comparisons}

This subsection compares the performance of different schemes in terms of the throughput and the outage probability.

Remark 2. Let $M<N$. The spatial diversity gained in the proposed CoMP-HARQ scheme with slow-fading channel is the same as the time diversity achieved in the non-CoMP-HARQ schemes when the channel is fastfading, i.e., when the channel gains change in each retransmission round independently.

In order to show this, consider a non-CoMP model where each user is always served by a specific BS and there is no cooperation between the BSs. In this way, the link between the $i$-th user and its corresponding BS is an interference-affected SISO channel. Assuming a fastfading model, i.e., the channels take new values in each retransmission round, the received SINR of the $i$-th user in round $n$ is $u(n)=\frac{P g_{i}(n)}{1+P \sum_{j=1 \neq i}^{N} g_{j}(n)}$ where $g_{i}(n)$ represents the gain realization of the channel between the $i$-th user and its corresponding BS at round $n$. Hence, a user throughput in the presence of the RTD and INR protocols is

$$
\eta_{i}^{\mathrm{RTD}, \mathrm{Fast}}=\sum_{m=1}^{M+1} \frac{R}{m} \operatorname{Pr}\left\{\log \left(1+\sum_{n=1}^{m-1} u(n)\right)<R \leq \log \left(1+\sum_{n=1}^{m} u(n)\right)\right\}
$$

$$
\eta_{i}^{\mathrm{INR}, \text { Fast }}=\sum_{m=1}^{M+1} \frac{R}{m} \operatorname{Pr}\left\{\sum_{n=1}^{m-1} \log (1+u(n))<R \leq \sum_{n=1}^{m} \log (1+u(n))\right\},
$$

respectively, which are the corresponding throughputs obtained for the $i$-th user in the proposed CoMP model when the channel is slow-fading (see (6) and (10)).

In other words, although each channel remains constant in all retransmission rounds of the slow-fading model, by switching between the BSs, a different SINR realization is observed in each round, the same as in the fast-fading channels. Thus, the CoMP model works well as (1) the slow-fading behavior of the channel gives the opportunity to accurately estimate the channels at the receivers and (2) the same diversity as in the fast-fading channels is gained by a simple cooperation approach. Finally, the spatial diversity exploited by the proposed CoMP scheme will be less than the time diversity achieved in the non-CoMP fast-fading channel if $M \geq N$. This is because it may occur that we return back to the same BSs when the number of retransmissions exceeds the number of BSs. However, this case is of less interest because the maximum number 
of possible HARQ-based retransmissions is normally less than the number of cooperative BSs.

Remark 3. It has been previously shown that the INR outperforms the RTD in terms of outage probability and throughput ([38], lemma 1). However, as $\log (1+x) \rightarrow x$ for small $x$ 's, (7) and (11) can both be rewritten as

$$
\eta=N \sum_{m=1}^{M+1} \frac{R}{m} \operatorname{Pr}\left\{\gamma^{(m-1)}<R \leq \gamma^{(m)}\right\}
$$

when $P \rightarrow 0$. That is, the same performance is achieved by the INR and RTD protocols at low transmission powers.

This is interesting when we remember that the superiority of the INR over the RTD is at the cost of complexity; in the INR, the codewords are changed in each retransmission which results in more complex encoders and decoders. Therefore, compared to the INR, the RTD is preferable at low transmission powers, because the same throughput and outage probability are achieved in both schemes while the RTD leads to less implementation complexity.

As demonstrated in [58], the gain of the INR scheme over the RTD increases with the initial transmission rate $R$. Also, [58] has previously shown that the difference between the performance of the RTD and INR protocols decreases with the SINR variation between the retransmissions. Thus, compared to the non-CoMP setup, the gain of the INR over RTD decreases in the CoMP scenario.

Finally, it is worth noting that the considered HARQ protocols, RTD and INR, lead to better system performance compared to (1) the open-loop communication model, (2) basic ARQ protocols, and (3) the case when repetition codes are implemented in a cooperative fashion; the open-loop communication model is a special case of the HARQ-based schemes with no retransmissions, i.e., $M=0$. Hence, setting $M=0$ in, e.g., (8), the open-loop system throughput is

$$
\eta^{\text {Open }}=N R\left(1-F_{\gamma^{1}}\left(e^{R}-1\right)\right)
$$

which is clearly less than the throughput obtained in (7) and (11). In basic ARQ, on the other hand, the same codeword is transmitted in different retransmission rounds and the users decode the data in each round independently of the previously received signals. In this way, with some manipulations, the throughput of the basic ARQ approach is found as

$$
\eta^{\text {Basic }}=N \sum_{m=1}^{M+1}\left(\frac{R}{m}\left(\prod_{l=1}^{m-1} \operatorname{Pr}\left\{\log \left(1+\gamma^{l}\right)<R\right\}\right) \operatorname{Pr}\left\{R \leq \log \left(1+\gamma^{m}\right)\right\}\right) .
$$

Then, as less information is exploited by the basic ARQ decoder, compared to the RTD, the throughput in the
RTD model is obviously higher than the throughput in the basic ARQ (the superiority of the HARQ protocols over the basic ARQ has been previously shown in the literature, e.g., [36,38]). Here, it is interesting to note that with a slow-fading condition, e.g., [38] has shown that there are no performance gains with basic ARQ and the optimal throughput/outage probability achieved by the basic ARQ is the same as the one in the open-loop communication setup if the channel does not change in the retransmissions. However, the proposed CoMP-HARQ approach makes it possible to utilize the SINR variations and, depending on the channel pdf, increase the throughput by implementation of basic ARQ.

Finally, using repetition codes the same codeword is transmitted by the switching BSs in different rounds, the same as in RTD. However, as opposed to RTD, the MRCbased decoder is not implemented in each round but only when all possible repetition rounds are used. In this case, removing the $m$-th, $m=1 \ldots M$, probability terms of (7), the system throughput for the repetition codes is obtained by

$$
\eta^{\mathrm{Rept}}=\frac{N R}{M+1} \operatorname{Pr}\left\{R \leq \log \left(1+\gamma^{(M+1)}\right)\right\}<\eta^{\mathrm{RTD}} \leq \eta^{\mathrm{INR}}
$$

The same arguments can be used when comparing the outage probability of these schemes. Here, the only important difference is that the RTD HARQ and the repetition code schemes lead to the same outage probability. This is because in RTD, the outage occurs if and only if the data is not decodable in the last retransmission round, the same as in the repetition codes.

\section{Simulation results}

In Figures $1,2,3,4$, and 5 , we set $\lambda_{i, j}=1, \forall i, j$, i.e., the channel is assumed to be homogenous. Figures 6 and 7 study the heterogenous networks. The throughput is given in npcu and transmission power is presented in decibels which is given by $10 \log _{10} P$ (as the noise variance is set to 1 , the transmission power is the same as the transmission SNR). For the outage probability, the results are obtained for fixed initial transmission rates, specified in the figures. For the throughput and in each transmission power, the initial transmission rate is optimized by exhaustive search, to maximize the throughput for that power.

Considering $N=4 \mathrm{BSs}$ and a maximum of $M=1$ retransmission round, i.e., a maximum of two (re)transmissions, Figure 1 demonstrates the system throughput for different schemes. As seen in the figure, the difference between the INR and RTD protocols is negligible at low transmission powers. Also, the system throughput starts to become bounded at high powers, in harmony 


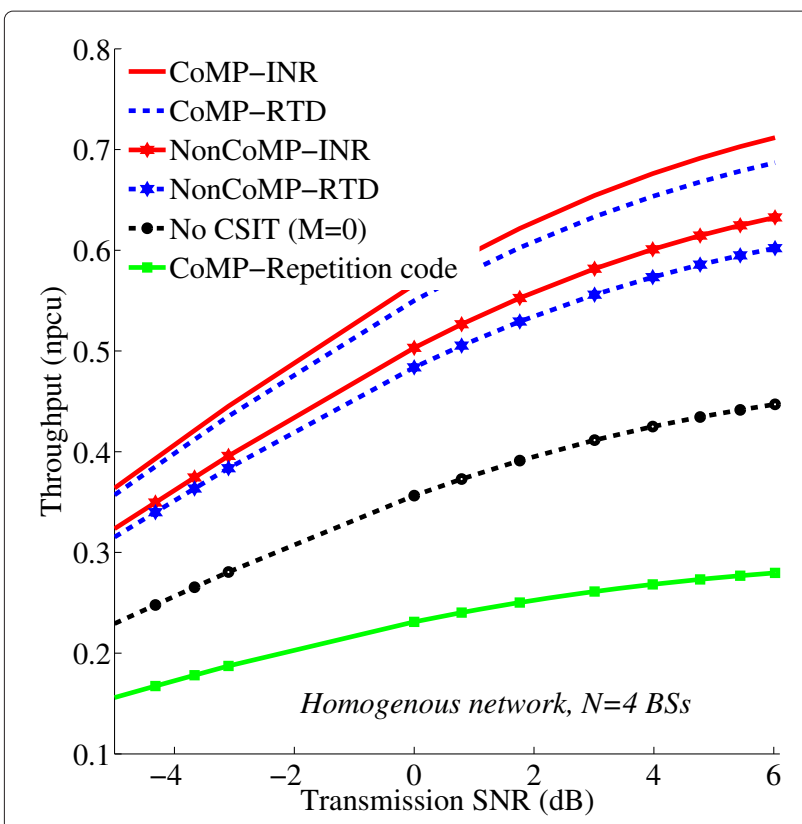

Figure 1 System throughput vs the transmission SNR $10 \log _{10} P$. Homogenous network $\lambda=1, N=4 \mathrm{BSs}$, a maximum of $M=1$ retransmission rounds for the HARQ protocols.

with Theorem 1. Therefore, implementation of HARQ in CoMP networks is more appropriate at low transmission powers. With even a single (re)transmission round, substantial performance improvement is achieved by the HARQ schemes, compared to the case with no CSI at the BSs. Also, the CoMP data transmission scheme results in considerable throughput increment, compared to the

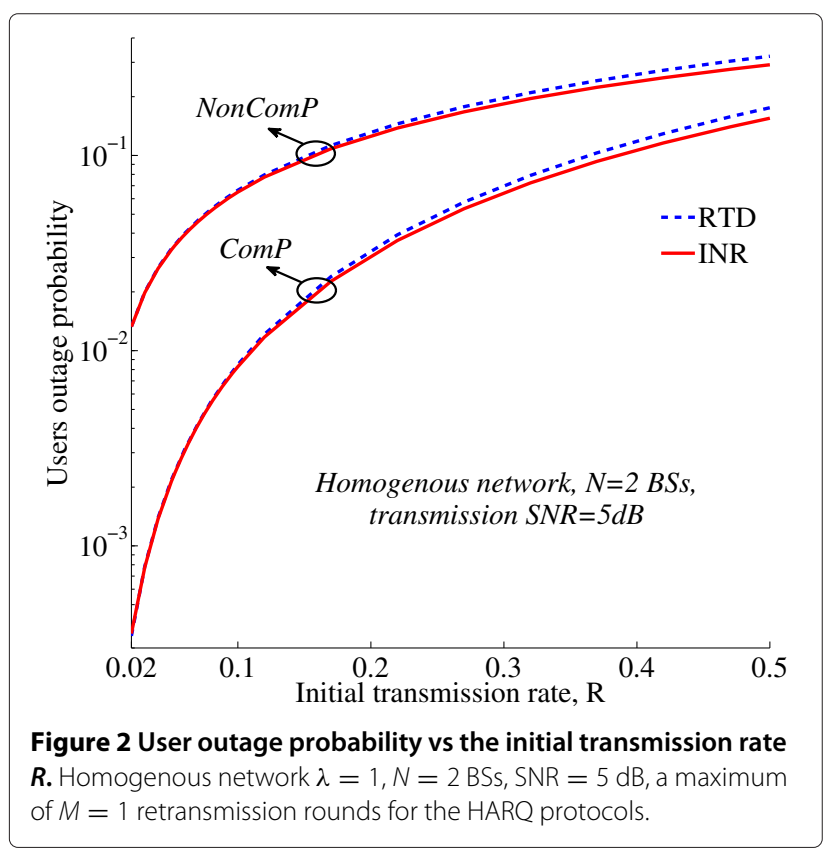

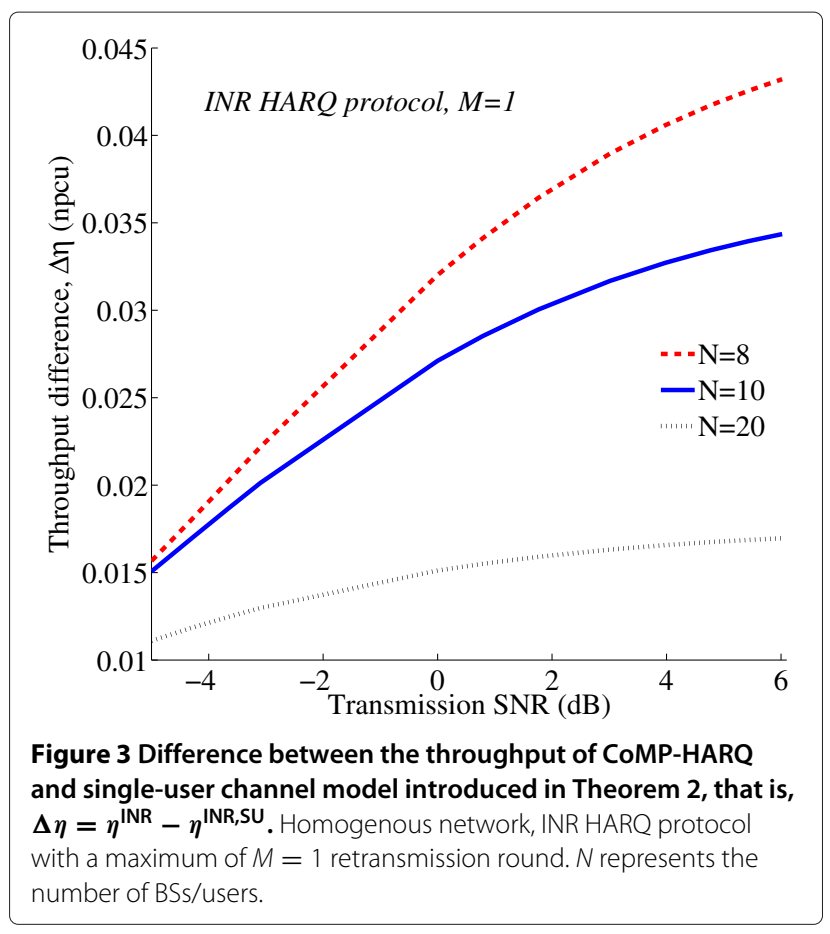

non-CoMP models. Interestingly, the cooperation gain, i.e., the difference between the throughput of the CoMP and non-CoMP schemes, is observed to be (almost) constant, at medium and high powers. Finally, the repetition codes are simple but perform poorly in terms of throughput, in comparison to the other schemes.

The effect of CoMP transmission on the user outage probability is studied in Figure 2 where, setting $N=2$, the user outage probability is obtained for different initial

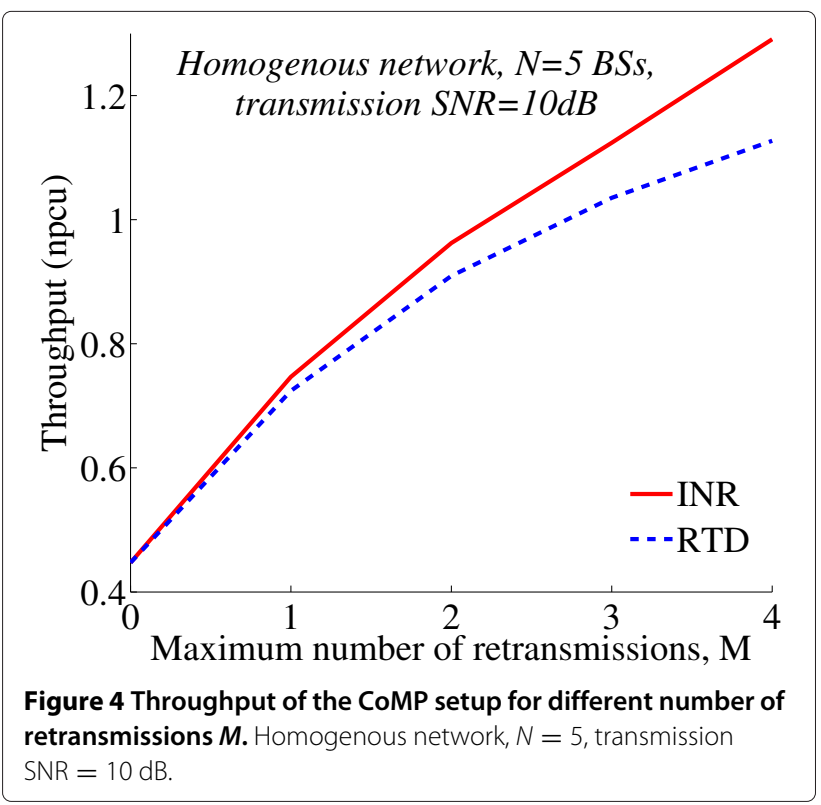




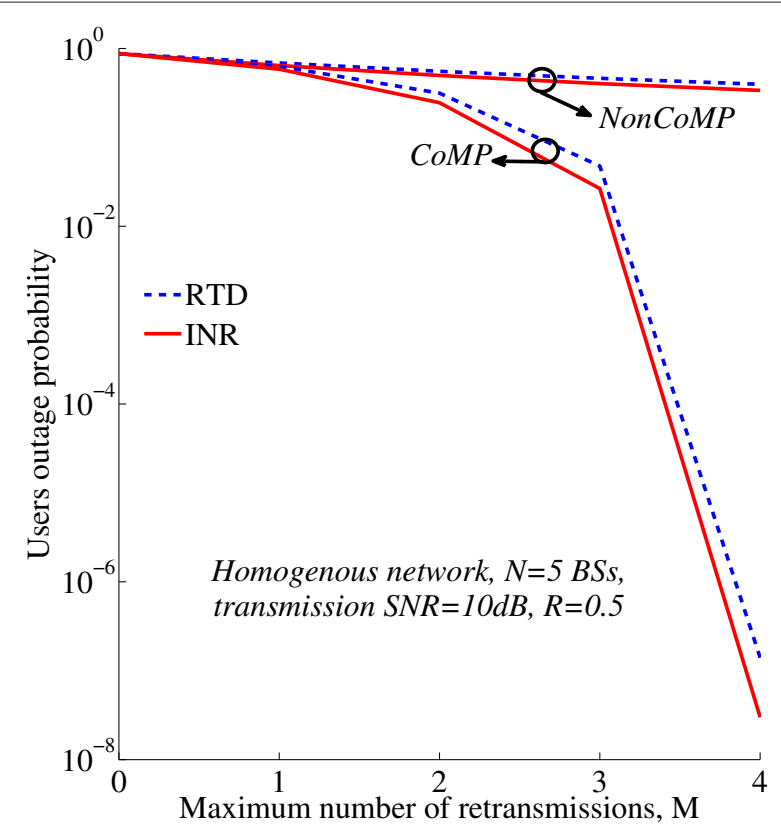

Figure 5 Outage probability for different number of retransmissions $\boldsymbol{M}$. Homogenous network, $N=5$, transmission $\mathrm{SNR}=10 \mathrm{~dB}$, initial transmission rate $R=0.5$.

transmission rates. As shown in the figure, CoMP transmission leads to considerable outage probability reduction, particularly at low rates. Moreover, the difference between the performance of the RTD and INR protocols increases with the initial transmission rate.

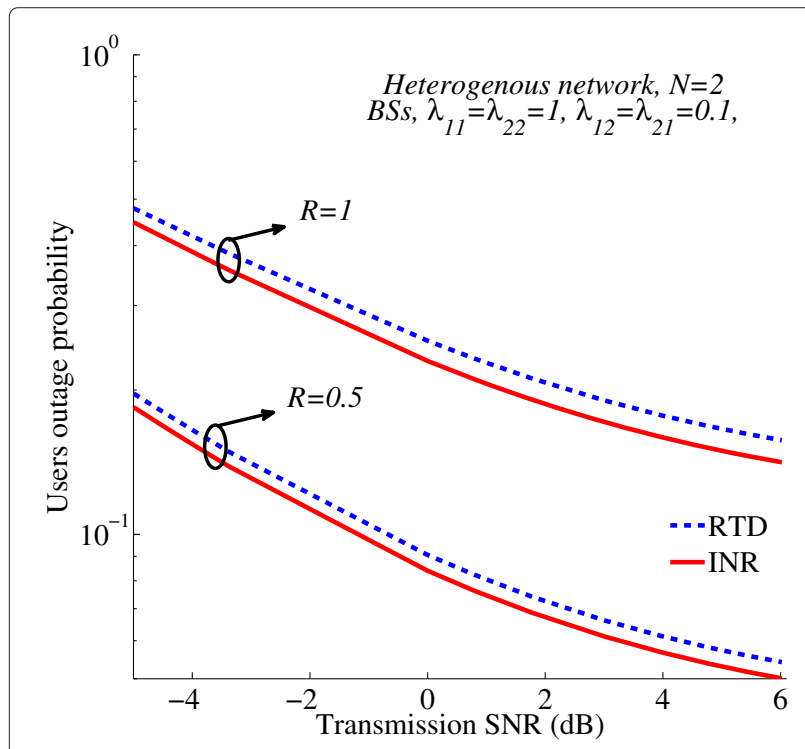

Figure 6 Outage probability vs the transmission SNR $10 \log _{10} P$. Heterogenous network $N=2$, a maximum of $M=1$ retransmission rounds. The fading parameters are set to $\lambda_{1,1}=\lambda_{2,2}=1$, $\lambda_{1,2}=\lambda_{2,1}=0.1$. The same outage probability as in the RTD scheme is achieved by repetition codes with repetition order of 2 .

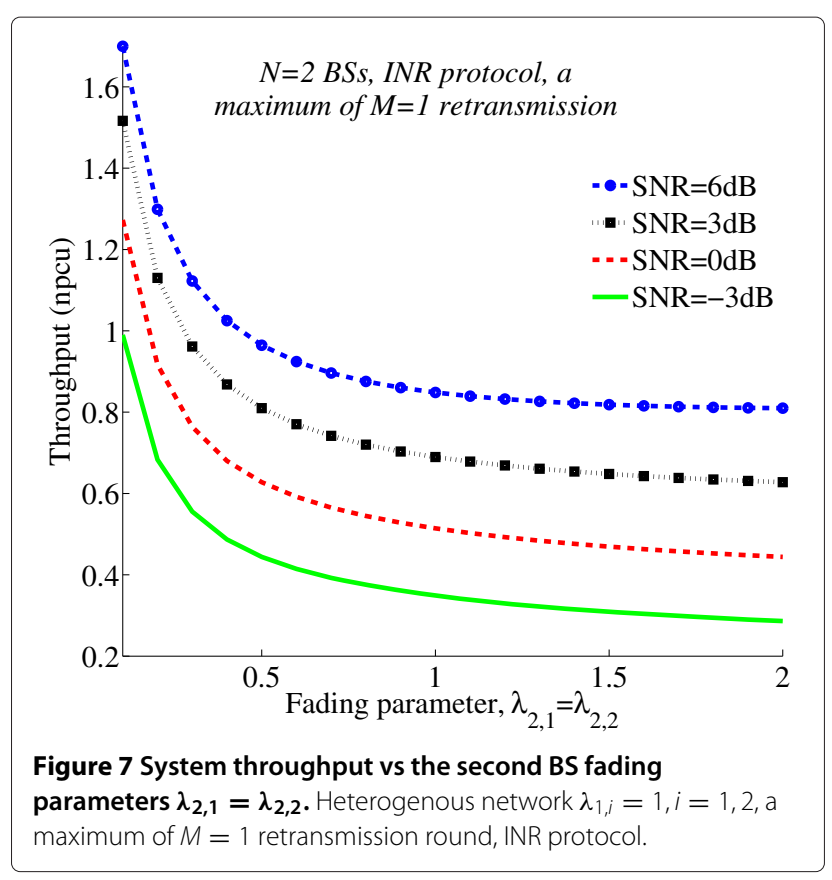

The validity of Theorem 2 is verified in Figure 3 . Here, the throughput difference $\Delta \eta=\eta^{\mathrm{INR}}-\eta^{\mathrm{INR}, \mathrm{SU}}$ is plotted as a function of the transmission power where $\eta^{\text {INR }}$ and $\eta^{\mathrm{INR}, \mathrm{SU}}$ are obtained by (11) and (20), respectively. As demonstrated in the figure, the difference between the throughput of the two models considered in Theorem 2 becomes small as the number of users increases. Also, the throughput difference increases with the transmission power. However, using (4) and (18), it can be shown that the difference becomes bounded as the power increases.

Setting $N=5$, Figures 4 and 5 study the system throughput and outage probability as a function of the maximum number of retransmissions. As demonstrated, considerable performance improvement is achieved by increasing the number of retransmissions. Also, the difference between the performance of INR and RTD protocols and the difference between the CoMP and non-CoMP data transmission models increase with $M$ substantially.

Figures 6 and 7 focus on heterogenous networks. With $N=2$ BSs and a maximum of $M=1$ retransmissions, Figure 6 shows a user outage probability for different transmission powers and initial transmission rates. Here, the fading parameters are set to $\lambda_{i, i}=1, i=1,2$, and $\lambda_{j, i}=0.1, i \neq j, i, j=1,2$. Again, the results show that the INR protocol outperforms the RTD, particularly at high transmission rates. However, as stated before, the superiority of the INR over RTD is at the cost of complexity at the encoders and decoders. Moreover, it is worth noting that, with the same argument as in Theorem 2, the user 
outage probability becomes bounded at high powers, as the system becomes interference-limited.

Finally, setting $N=2$ and $M=1$, Figure 7 investigates the system throughput in a heterogenous network. Here, while we set $\lambda_{1, i}=1, i=1,2$, the throughput is plotted as a function of the fading coefficients associated with the second BS, i.e., $\lambda_{2,1}=\lambda_{2,2}$. As it can be seen, the system throughput decreases when the distance between the second BS and the users, modeled by $\lambda_{2, i}, i=1,2$, increases. At asymptotic condition $\lambda_{2, i} \rightarrow \infty, i=1,2$, the network is mapped to a $1 \times 2$ SIMO channel working in a TDMA fashion.

\section{Conclusions}

This paper studied the performance of a CoMP-HARQ network. The proposed approach exploits the advantages of coordinated data transmission schemes and, meanwhile, solves some of the important problems that may limit the practical implementation of CoMP networks. As demonstrated both theoretically and numerically, considerable performance improvement can be achieved by implementation of CoMP-HARQ systems. However, the system performance becomes bounded if the number of HARQ-based retransmission rounds does not scale with the transmission power. The proposed CoMP network can be modeled by a collection of interference-free single-user channels experiencing a specific SNR. Finally, the diversity combining HARQ schemes outperform the code-combining HARQ protocols at low transmission powers. This is because the same outage probability and throughput are achieved by these protocols, while the diversity combining schemes lead to less implementation complexity.

\section{Endnotes}

${ }^{\mathrm{a} A}$ packet is defined as the transmission of a codeword along with all its possible retransmission rounds.

${ }^{\mathrm{b}}$ This is the best scheme for scheduling $N$ users with no CSIT at the BSs [56] as well as when, due to scheduling delay and complexity, the users are selected for transmission based upon queue lengths instead of on channel conditions.

${ }^{\mathrm{c}}$ For heterogenous Rayleigh fading channels, $f_{g_{j, i}}(g)=\lambda_{j, i} e^{-\lambda_{j, i} g}, g \geq 0$, (4) is rephrased as $F_{\gamma_{k, i}^{m}}(x)=1-\frac{e^{-\frac{\lambda_{k+m-1, i}}{P}}}{\prod_{j=1, j \neq m}^{N}\left(1+\frac{\lambda_{k+m-1, i}}{\lambda_{k+j, i}} x\right)}, x \geq 0$.

${ }^{\mathrm{d}}$ Straightforward modifications can be applied to (13) and (14) to prove the theorem for heterogenous networks.

${ }^{\mathrm{e}}$ The random variable $X$ dominates the random variable $Y$ if $F_{X}(x) \geq F_{Y}(x), \forall x$ [59].

${ }^{\mathrm{f}}$ For heterogenous channels, we can use $F_{\gamma_{k, i}^{m}}(x) \leq$ $F_{\omega_{k, i}^{m}}(x), \forall x$, where $F_{\omega_{k, i}^{m}}=1-e^{-\left(\frac{\lambda_{k+m-1, i}}{P}+\sum_{j=1, j \neq m}^{N} \frac{\lambda_{k+m-1, i}}{\lambda_{k+j, i}}\right) x}$.

\section{Competing interests}

The authors declare that they have no competing interests.

Received: 25 February 2013 Accepted: 2 August 2013 Published: 16 August 2013

\section{References}

1. D Gesbert, S Hanly, H Huang, S Shamai, O Simeone, W Yu, Multi-cell MIMO cooperative networks: a new look at interference. IEEE J. Sel. Areas Commun. 28(9), 1380-1408 (2010)

2. J Zhang, Networked MIMO with clustered linear precoding. IEEE Trans. Wireless Commun. 8(4), 1910-1921 (2009)

3. CB Chae, S Kim, RW Heath, Network coordinated beamforming for cell-boundary users: linear and nonlinear approaches. IEEE J. Sel. Topics Signal Process. 3(6), 1094-1105 (2009)

4. C Shen, TH Chang, KY Wang, Z Qiu, CY Chi, Distributed robust multi-cell coordinated beamforming with imperfect CSI: an ADMM approach. IEEE Trans. Signal Process. 60(6), 2988-3003 (2012)

5. P Marsch, G Fettweis, in ICC 2007. A framework for optimizing the uplink performance of distributed antenna systems under a constrained backhaul (Glasgow, 24-28 June 2007), pp. 975-979

6. A Sanderovich, O Somekh, S Shamai, in ISIT 2007. Uplink macro diversity with limited backhaul capacity (Nice, 24-29 June 2007), pp. 11-15

7. BL Ng, JS Evans, SV Hanly, D Aktas, Distributed downlink beamforming with cooperative base stations. IEEE Trans. Inf. Theory. 54(12), 5491-5499 (2008)

8. N Levy, S Shamai, Clustered local decoding for wyner-type cellular models. IEEE Trans. Inf. Theory. 55(11), 4967-4985 (2009)

9. N Levy, S Shamai, Information theoretic aspects of users' activity in a wyner-like cellular model. IEEE Trans. Inf. Theory. 56(5), 2241-2248 (2010)

10. MK Karakayali, GJ Foschini, RA Valenzuela, Network coordination for spectrally efficient communications in cellular systems. IEEE Wireless Commun. 13(4), 56-61 (2006)

11. S Shamai, O Simeone, O Somekh, A Sanderovich, BM Zaidel, HV Poor, in PIMRC 2008. Information-theoretic implications of constrained cooperation in simple cellular models (Cannes, 15-18 September 2008), pp. 1-5

12. S Shamai, O Simeone, O Somekh, HV Poor, in ITAW 2008. Joint multi-cell processing for downlink channels with limited-capacity backhaul (Washington, D.C., 2-4 June 2008), pp. 345-349

13. J Hoydis, M Kobayashi, M Debbah, Optimal channel training in uplink network MIMO systems. IEEE Trans. Signal Process. 59(6), 2824-2833 (2011)

14. P Marsch, G Fettweis, in GLOBECOM 2009. On downlink network MIMO under a constrained backhaul and imperfect channel knowledge (Honolulu, 30 November-4 December 2009), pp. 1-6

15. TWild, in VTC 2011. Comparing downlink coordinated multi-point schemes with imperfect channel knowledge (Budapest, 15-18 May 2011), pp. $1-5$

16. H Sun, X Zhang, W Fang, in PIMRC 2011. Dynamic cell clustering design for realistic coordinated multipoint downlink transmission (Toronto, 11-14 September 2011), pp. 1331-1335

17. B Ghimire, G Auer, H Haas, Busy burst enabled coordinated multipoint network with decentralized control. IEEE Trans. Wireless Commun. 10(10), 3310-3320 (2011)

18. J Shi, T Zhang, Y Zhou, Z Zeng, Z Huang, in VTC 2011. Rate loss caused by limited feedback and channel delay in coordinated multi-point system (Budapest, 15-18 May 2011), pp. 1-6

19. X Hou, C Yang, in ICASSP 2011. How much feedback overhead is required for base station cooperative transmission to outperform non-cooperative transmission? (Prague, 22-27 May 2011), pp. 3416-3419

20. M Eslami, WA Krzymien, M Al-Shalash, in MC-SS 2011. A hybrid mode user-location-aware network MIMO with limited feedback for cellular downlink (Herrsching, 3-4 May 2011), pp. 1-4

21. F Yuan, C Yang, Bit allocation between per-cell codebook and phase ambiguity quantization for limited feedback coordinated multi-point transmission systems. IEEE Trans. Commun. 60(9), 2546-2559 (2012)

22. Y Li, J Ge, C Shen, J Li, W Miao, in ISCIT 2011. Coordinated multi-point transmission with limited feedback for LTE-advanced (Hangzhou, 12-14 October 2011), pp. 68-72 
23. 3GPP TS 36.211 v9.1.0, Evolved universal terrestrial radio access (E-UTRA); physical channels and modulation. March 2010

24. J Jin, Q Wang, C Lin, H Yang, Y Wang, in GLOBECOM 2010. Coordinated multi-point transmission with limited feedback (Miami, 6-10 December 2010), pp. 850-854

25. TR Lakshmana, A Papadogiannis, J Li, T Svensson, in PIMRC 2012. On the potential of broadcast CSI for opportunistic coordinated multi-point transmission (Sydney, 9-12 September 2012), pp. 1262-1267

26. J Li, A Papadogiannis, R Apelfrojd, T Svensson, M Sternad, in PIMRC 2012. Performance evaluation of coordinated multi-point transmission schemes with predicted CSI (Sydney, 9-12 September 2012), pp. 1055-1060

27. D Kim, OS Shin, I Sohn, KB Lee, Channel feedback optimization for network MIMO systems. IEEE Trans. Veh. Technol. 61(7), 3315-3321 (2012)

28. D Kim, OS Shin, KB Lee, in GLOBECOM 2011. Efficient limited feedback schemes for network MIMO systems (Houston, TX, 5-9 December 2011), pp. 1-6

29. S Zhou, J Gong, Z Niu, Distributed adaptation of quantized feedback for downlink network MIMO systems. IEEE Trans. Wireless Commun. 10(1), 61-67 (2011)

30. F Yuan, C Yang, in VTC 2011. Phase ambiguity quantization for per-cell codebook based limited feedback coordinated multi-point transmission systems (San Francisco, 5-8 September 2011), pp. 1-5

31. MR Khanzadi, H Mehrpouyan, E Alpman, T Svensson, D Kuylenstierna, T Eriksson, in ICSPCS 2011. On models, bounds, and estimation algorithms for time-varying phase noise (Honolulu, 12-14 December 2011), pp. 1-8

32. F Munier, T Eriksson, A Svensson, An ICl reduction scheme for OFDM system with phase noise over fading channels. IEEE Trans. Commun. 56(7), 1119-1126 (2008)

33. F Peng, J Zhang, WE Ryan, in WCNC 2007. Adaptive modulation and coding for IEEE 802.11n (Hong Kong, 11-15 March 2007), pp. 656-661

34. WiMAX Forum, Mobile WiMAX - part Il: a comparative analysis. (WiMAX, Solana Beach, 2006)

35. 3GGP, LTE. http://www.3gpp.org/LTE

36. G Caire, D Tuninetti, The throughput of hybrid-ARQ protocols for the Gaussian collision channel. IEEE Trans. Inf. Theory. 47(5), 1971-1988 (2001)

37. B Makki, T Eriksson, On hybrid ARQ and quantized CSI feedback schemes in quasi-static fading channels. IEEE Trans. Commun. 60(4), 986-997 (2012)

38. C Shen, T Liu, MP Fitz, On the average rate performance of hybrid-ARQ in quasi-static fading channels. IEEE Trans. Commun. 57(11), 3339-3352 (2009)

39. B Makki, T Eriksson, On the average rate of HARQ-based quasi-static spectrum sharing networks. IEEE Trans. Wireless Commun. 11(1), 65-77 (2012)

40. B Makki, A Graell i Amat, T Eriksson, in WCNC 2012. Power allocation in repetition time diversity hybrid automatic repeat request feedback (Paris, 1-4 April 2012), pp. 2329-2334

41. B Makki, A Graell i Amat, T Eriksson, HARQ feedback in spectrum sharing networks. IEEE Commun. Lett. 16(9), 1337-1340 (2012)

42. HE Gamal, G Caire, MO Damen, The MIMO ARQ channel: diversity-multiplexing-delay tradeoff. IEEE Trans. Inf. Theory. 52(8), 3601-3621 (2006)

43. THolliday, AJ Goldsmith, HV Poor, Joint source and channel coding for MIMO systems: Is it better to be robust or quick? IEEE Trans. Inf. Theory. 54(4), 1393-1405 (2008)

44. A Chuang, A Guillen, i Fabregas, LK Rasmussen, IB Collings, Optimal throughput-diversity-delay tradeoff in MIMO ARQ block-fading channels. IEEE Trans. Inf. Theory. 54(9), 3968-3986 (2008)

45. KD Nguyen, LK Rasmussen, A Fabregas, N Letzepis, in AusCTW 2009 Rate-diversity-delay tradeoff for ARQ systems over MIMO block-fading channels (Sydney, 4-7 February 2009), pp. 116-121

46. H Liu, L Razoumov, N Mandayam, P Spasojevic, An optimal power allocation scheme for the STC hybrid-ARQ over energy limited networks. IEEE Trans. Wireless Commun. 8(12), 5718-5722 (2009)

47. KD Nguyen, LK Rasmussen, A Guillen, i Fabregas, N Letzepis, MIMO ARO with multibit feedback: outage analysis. IEEE Trans. Inf. Theory. 58(2), 765-779 (2012)

48. Y Gao, Y Li, H Yu, S Gao, X Wang, in CHINACOM 2011. Modeling and analysis of SU-COMP HARQ in 3GPP LTE system level simulation (Harbin, 17-19 August 2011), pp. 295-298

49. 3GPP, TR25.835, Report on Hybrid ARQ Type II/II[s]. http://www.3gpp. $\mathrm{org} / \mathrm{ftp} /$ Specs/html-info/25835.htm
50. HL Määttänen, K Hämäläinen, J Venäläinen, K Schober, M Enescu, M Valkama, System-level performance of Ite-advanced with joint transmission and dynamic point selection schemes. Eurasip J. Adv. Signal Process (2012)

51. PartnershipProject 3rd Generation, TSG RAN: (E-UTRA); 3GPP TS 36.213 version 11.0.0 Physical layer procedures (Release 11) (2012). http://www 3gpp.org/

52. S Wicker, Error Control Systems for Digital Communication and Storage. (Prentice-Hall, Upper Saddle River, 1995)

53. TM Cover, JA Thomas, Elements of Information Theory. (Wiley, New Jersey, 2006)

54. S Sesia, G Caire, G Vivier, Incremental redundancy hybrid ARQ schemes based on low-density parity-check codes. IEEE Trans. Commun. 52(8), 1311-1321 (2004)

55. IN Bronshtein, M van den Brand, Handbook of Mathematics, 5th ed. (Springer-Verlag, Berlin Heidelberg, 2007)

56. N Jindal, MIMO broadcast channels with finite-rate feedback. IEEE Trans. Inf. Theory. 52(11), 5045-5060 (2006)

57. E Telatar, Capacity of multi-antenna gaussian channels. Eur Trans. Telecommun. 10, 585-595 (1999)

58. J-F Cheng, Coding performance of hybrid ARQ schemes. IEEE Trans. Commun. 54(6), 1017-1029 (2006)

59. VS Bawa, Optimal rules for ordering uncertain prospects. J. Financ. Econ. 2(1), 95-121 (1975)

doi:10.1186/1687-1499-2013-209

Cite this article as: Makki et al:: On an HARQ-based coordinated multipoint network using dynamic point selection. EURASIP Journal on Wireless Communications and Networking 2013 2013:209.

\section{Submit your manuscript to a SpringerOpen ${ }^{\circ}$ journal and benefit from:}

- Convenient online submission

- Rigorous peer review

- Immediate publication on acceptance

- Open access: articles freely available online

- High visibility within the field

- Retaining the copyright to your article

Submit your next manuscript at $>$ springeropen.com 